\title{
Use of Selective Serotonin Reuptake Inhibitors During Pregnancy and Prevalence of Congenital Malformations: A Protocol for Systematic Review and Meta-analysis of Observational Studies from 2009-2020.
}

\section{Ayodele Lucy Fela-Thomas}

Federal Neuro Psychiatric Hospital

Emmanuel Okechukwu Nna ( $\nabla$ e.nna@themping.org )

The Molecular Pathology Institute, Enugu https://orcid.org/0000-0001-6791-2336

Nnaemeka Anyahara

: General Hospital Lagos

Oluwafemi T Ojo

Lagos State University Teaching Hospital

Olugbemi Motilewa

University of Uyo Teaching Hospital

\section{Emeka E Okoh}

Nnamdi Azikiwe University Teaching Hospital

Micheal A Alao

University College Hospital Ibadan

Umar A Kaoje

Usmanu Danfodiyo University College of Health Sciences

\section{Ogugua Osi-Ogbu}

National Hospital Abuja

Chinaza Richard Ikeagwulonu

Alex Ekwueme Federal University Teaching Hospital Abakaliki

Ifeoma Joy Okoye

University of Nigeria Teaching Hospital

\section{Protocol}

Keywords: Pregnancy, trimester, depression, antidepressants, selective serotonin reuptake inhibitors, SSRI, offspring, congenital malformation 
Posted Date: November 9th, 2020

DOI: https://doi.org/10.21203/rs.3.rs-102876/v1

License: (c) (i) This work is licensed under a Creative Commons Attribution 4.0 International License. Read Full License 


\section{Abstract}

\section{Background:}

The effect of antidepressants on the foetus is of great public concern on account of teratogenicity. However, evidence on this is quite controversial. This study aims to determine the risk of congenital malformations in offsprings of pregnant women placed on Selective Serotonin Reuptake Inhibitors (SSRIs) during pregnancy compared to the offsprings of pregnant women not on SSRIs. It also aims to assess if significant increase in risk occurs across trimesters.

\section{METHODS AND ANALYSIS:}

A search strategy is developed using MeSH, text words and entry terms. Nine databases will be searched: PubMed, Embase, CINAHL, AJOL, Google Scholar, Web of Science, Cochrane Library, Research gate and Scopus. Only Observational studies from 2009-2020, retrievable in the English Language will be included. The primary measurable outcome is risk of teratogenicity with SSRI use in pregnancy. All identified studies, imported into End note version 9, will be screened based on the inclusion/exclusion criteria; data will be exported into Microsoft excel. Extractable data will include first author's name and year of publication, proportion of congenital malformations in women on SSRIs and women without SSRIs, trimester, time of initiation and duration of treatment. Pedro quality scores and Cochrane risk of bias for individual study will be reported. All studies will be assessed for methodological, clinical and statistical heterogeneity. Publication bias will be assessed using the funnel plot. Subgroup analysis will be performed. The different trimesters and time of initiation of treatment will be used as moderators. Metaregression will be done using duration of treatment. The CMA version 3 will be used for statistical analysis and forest plots.

\section{Discussion:}

The study will require no ethical approval, it is based on published data. The results obtained from this review will provide relevant information on the use of SSRIs in pregnancy and the risk of congenital malformations. Furthermore, it will provide vital information on the levels and trend of risk at each trimester. The final report will be made available to mental health experts providing care to pregnant women.

\section{Trial Registration Number.}

This protocol is registered in PROSPERO, with number CRD42020213505

\section{Background}

The prevalence of depression in pregnancy ranges from 4-20\%. Major depression affects about $12 \%$ of pregnant women (1). A population based prospective study conducted among some pregnant women in Oslo, Norway found a prevalence of 8.6\% among Western European women, 19.5\% in Middle Easterner 
pregnant women, $17.5 \%$ South Asians and $11.3 \%$ among women of other groups (2). A prevalence of $47 \%$ was reported in a study conducted among pregnant women attending some primary care clinics in South Africa (3). A study conducted at some antenatal clinics in South Western Nigeria reported a prevalence of $24.5 \%$ for ante natal depression among the pregnant women (4).

Risk factors for depression in pregnancy include a previous history of depression, economic deprivation, poor social support, single marital status, young maternal age, domestic violence, smoking and obstetric factors like gravidity, unplanned pregnancy, previous termination of pregnancy and history of obstetric complications (4-8). Depression in pregnancy is associated with complications both for mother and the unborn child $(2,9-11)$. A prospective cohort study conducted in the UK among pregnant women found that ante natal depression was a strong risk factor for depression in their off springs at age 18 years(12). Untreated maternal depression has been linked to increased suicide risk and risk of postpartum depression in the mother including the risk of miscarriage and preterm births (13). Furthermore, untreated maternal depression is reported to be associated with detrimental effects such as hyperactivity, altered foetal heart rate on the growing foetus, deranged cortisol, dopamine and norepinephrine levels, stress or depressive- like behaviours and premature deaths in the new born, while in the children, it is said to be associated with internalizing and externalizing behaviour (14).Thus, it is imperative that proper intervention is given to such patients depending on the severity of symptoms.

Treatment of depression in pregnancy mirrors the treatment of depression in non- pregnant women. Most guidelines support psychotherapy as first line treatment for mild and moderate depression, with special attention to individual characteristics. However, most guidelines advocate treatment with pharmacotherapy for severe depression and in some instances of moderate depression (15). The use of antidepressants in pregnancy has been associated with poor perinatal and childhood outcomes $(16,17)$. The most prescribed antidepressants for maternal depression are the Selective Serotonin Reuptake Inhibitors (SSRIs)(18). However, current reports associate the use of this class of antidepressants with teratogenicity and poor outcome in children although evidence supporting this is inconclusive $(16,19-$ 21). For instance, in a nested cohort study among women who had been treated with antidepressants during pregnancy particularly during the first trimester, the authors observed no association between foetal cardiac defects and prenatal antidepressant use especially the SSRIs (22). But another study conducted in Denmark reported an increased risk of congenital malformations of the heart in children of mothers exposed to SSRI use in the first trimester of pregnancy(23).

\section{Method And Design}

Aim: this study aims to determine the pooled risk of congenital malformations in offsprings of pregnant women placed on SSRIs during pregnancy compared to the offsprings of pregnant women not on SSRIs. It secondarily aims to assess if significant increase in risk of congenital malformations occurs across the different trimesters and to determine if the trend has been on the increase or decline.

\section{Objectives:}


The specific objectives of the study are:

1. to determine the pooled risk of congenital malformations in offsprings of women placed on SSRIs during pregnancy.

2. to assess the level of risk of congenital malformations across trimesters.

3. to assess the trend of risk, if any, of congenital malformations over the years.

\section{Review Questions:}

1. What is the risk of congenital malformations in the offspring of women placed on SSRIs during pregnancy compared to offspring of women not placed on SSRIs during pregnancy?

2. Does the risk of teratogenicity if any, with SSRI use in pregnancy increase across the different trimesters?

3. Is there a trend of teratogenicity with SSRIs use in pregnancy over the years, 2009-2020?

\section{Design:}

This is a protocol for systematic review and meta-analysis on observational studies from 2009 to 2020 , focussing on the use of SSRIs in pregnancy and risk of congenital malformations. This review will look at the proportion of pregnancies in women on SSRIs that resulted in congenital malformations. It will also assess if the risk of congenital malformations occurs at the different trimesters and if there is an increase or a decrease in the trend of teratogenicity with SSRI use in pregnancy over the years.

\section{INCLUSION CRITERIA:}
A. Observational studies: Cohort studies, case control studies, cross sectional studies, historic cohort studies.
B. Studies must be published between 2009-2020.
C. Studies must be published and retrievable in English.
D. Studies reporting occurrence or risk of teratogenicity with SSRI use in pregnancy

\section{Exclusion criteria:}
a. Reviews, commentaries, letters to editors and editorials.
b. Studies reporting in- vitro and animal studies.
c. Interventional studies including randomized clinical trials and quasi-clinical trials.
d. Duplicates or replicates of studies.
e. Studies not retrievable in English. 
This review will be reported in line with Preferred Reporting Items for Systematic Reviews and MetaAnalyses (PRISMA 2015 Statement).

\section{Information sources}

The search will employ sensitive topic-based strategies designed for each database. The search will be carried out in the following databases: PUBMED, EMBASE, CINAHL, RESEARCHGATE, AJOL, GOOGLE SCHOLAR, WEB OF SCIENCE, SCOPUS and COCHRANE LIBRARY. Only observational studies retrievable in the English Language will be included.

\section{Search strategy}

This will include MESH terms, Text words and Entry terms. Table 1 illustrates the search strategies to be used in the databases.

\begin{tabular}{|lll|}
\hline S/No & Database & Search strategy \\
\hline 1 & PubMed & $\begin{array}{l}\text { ((")Pregnancy"[Mesh] OR Pregnancies OR Gestation) AND ((5-Hydroxytryptamine } \\
\text { Uptake Inhibitors OR 5 Hydroxytryptamine Uptake Inhibitors OR Selective } \\
\text { Serotonin Reuptake Inhibitors OR 5-HT Uptake Inhibitors OR 5 HT Uptake } \\
\text { Inhibitors) OR "Serotonin Uptake Inhibitors"[Mesh])) AND ((Congenital } \\
\text { Abnormality OR Congenital Defects OR Congenital Defects OR Birth Defects OR } \\
\text { Birth Defect) OR "Congenital Abnormalities"[Mesh]). Filters: From 2009 to 2020 }\end{array}$ \\
\hline \multicolumn{2}{|l|}{ The same search strategy will be used in other databases with slight modifications. } \\
\hline
\end{tabular}

\section{PICOs:}

Participants: In this study, the participants are pregnant women placed on SSRIs.

Intervention: SSRI,

Comparator: The Comparator is pregnant women not on SSRI.

Outcomes: the primary outcome is proportion of live births with congenital malformations. Secondary outcomes include risk of congenital malformation across the different trimesters.

\section{Data Extraction and Management}

\section{a. Data Extraction:}

Studies will be searched using the search strategy in Table 1. There are four levels of data screening in this study: 
a. Level 1 is based on the study design: only observational studies, published and/ or retrievable in the English Language will be included; other study designs will be excluded;

b. Level 2: selected studies will first be screened by titles and abstracts using entry terms, keywords, and MeSh terms;

c. level 3: selected studies will be further screened by full-text reading using the same strategy;

d. level 4: snowballing of literature from included studies.

Eleven reviewers are involved in this study. A pair of reviewers will independently screen studies from each database. Conflicts will be resolved by a third independent reviewer. The review will not be blinded. All screened and retrieved items will be exported to Endnote version 9. After screening, data will be exported to Microsoft Excel. All relevant studies that meet the inclusion criteria and are exported to Microsoft Excel will also have full texts retrieved and read to enable snowballing search on references. Authors of eligible studies with missing data will be contacted by email or telephone. Grey literature will not be included; only data available in electronic databases.

\section{b. Selection process:}

Two independent reviewers will screen full texts of all eligible observational studies and snowballed articles and conflicts will be resolved by a third reviewer.

\section{c. Data collection process:}

De-duplication of the studies will then be done in the EndNote version 9. Eligible studies will be exported and screened in Microsoft Excel. The following data will be extracted from the Microsoft Excel file:

a. First author's surname and year of publication;

b. prevalence or proportion of congenital malformations in women on SSRIs;

c. prevalence or proportion of congenital malformations in women not on SSRIs

d. sample size,

e. trimester and f) time of initiation of drug use and g) duration of drug use

Data from Excel will be exported to CMA Software for meta-analysis.

\section{Data items/Measurable outcomes}

Data items for statistical synthesis include the following:

a. proportion of pregnancies in women on SSRIs that resulted in congenital malformations (primary outcome). This is interpreted as a success event. It can also be reported as a relative risk. The effect size to be used for analysis is relative risk (RR). Other effect sizes from similar design and report will be converted to RR in the CMA Software. 
b. Proportion of pregnancies in women not on SSRI who had babies with congenital malformations. This is interpreted as a failure event. It will be considered in reporting of relative risk (RR) as effect size.

c. Trimester of pregnancy that has teratogenicity.

d. Time of initiation of drug use during pregnancy.

e. Duration of SSRI use in pregnancy.

\section{Risk of bias}

The risk of bias will be accessed for the individual studies using the National Institute of Health $(\mathrm{NIH})$ Quality assessment tool for observational cohort and cross-sectional studies. This will be cross-checked with the Cochrane tool of risk of bias assessment for the strength of the body of evidence; i.e. using specific relevant items from this tool to assess the strength of the body of evidence.

The following areas shall be assessed and any study with extreme bias will be excluded following consensus decision.

i. Method of reporting: this will be done at the outcome level.

ii. Reporting of study: Studies that were reported in different units but similar in outcome and design will be converted based on individual case evaluation. This will be evaluated for individual studies by assessing unit of reporting of studies, for example, whether prevalence with confidence intervals, OR or RR was reported. This will be done at the study level

iii. Heterogeneity will be assessed at the study level

iv. Publication bias will be assessed at the study level

\section{Data synthesis}

a. Studies that passed the methodological quality assessment using the NIH quality assessment tool will be extracted. The results will be presented in tabular format in addition to a narrative synthesis.

b. The following will be included into the meta-analysis;

1. Proportion of pregnant women on SSRIs and had babies with congenital malformation and proportion of pregnant women not on SSRIs who had babies with congenital abnormalities. Effect size is relative risk.

2. Quantitative analysis

Pooled RR of congenital malformations will be calculated with $95 \% \mathrm{Cl}$. This is the primary measurable outcome. 
Sub-group analysis using the different trimesters and time of initiation of drug use, as categorical data and moderators, will be performed.

Meta-regression on relative risk of congenital malformations using duration of drug use as a moderator (quantitative) will also be performed.

Cumulative meta-analysis be performed to check for trend in the RR of congenital malformation from 2009 to 2020.

\section{Discussion}

In addition to systematic review, data will be quantitatively analysed using the Comprehensive Meta Analysis software version 3(Biostat, USA). For each reported RR of congenital malformations, the standard error and variance for each specific study will be reported. Data summary will report pooled RR of congenital malformations in babies of women on SSRIs compared to those without SSRIs. Data analysis will seek to establish if use of SSRI in pregnancy results in increased risk of congenital abnormalities. It will discuss the level of risk across the trimesters, highlighting which trimester has the highest level of teratogenicity. It will also document any trend on RR of congenital malformations over the years, 2009 to 2020 .

\section{Assessment of Meta-bias}

The pooled relative risk and subgroup analysis will be presented using a forest plot. Heterogeneity will be assessed using the $Q$ statistics and its $p$ value, tau ${ }^{2}$ and the $I^{2}$. $I^{2}$ values of less than $40 \%$ will be considered low heterogeneity while values $>40$ but $<75 \%$ will be considered moderate and values $>75 \%$ are high. Sensivity analysis will be performed if heterogeneity is $>75 \%$.

Both random and fixed effect models will be assessed, and the appropriate model will be taken based on the forest plots. Publication bias in the selection of studies will be tested using funnel plot (trim and fill method) and test for funnel plot asymmetry.

\section{Confidence in Cumulative Evidence}

The study selection process will be summarized in a flow diagram according to the PRISMA 2015 Statement (24) and PRISMA-P Checklist (included in appendix). A table of search strategy in various databases showing text words, MeSH and entry terms will be included. List of included studies will be summarized in a table. Quantitative data such as pooled relative risk and $95 \% \mathrm{Cl}$, p values, relative weights assigned to studies and heterogeneity tests will be included in the forest plots. A table of quality scores and risk of bias of each eligible study will be included. Forest plots to show sub-group analysis, meta-regression and cumulative meta-analysis of 2009-2020 will be included. The final study will be published in a peer-review journal and the findings will be disseminated to relevant health authorities and clinical groups that manage pregnant women and newborns. 


\section{Abbreviations}

AGCPN: The Association for Good Clinical Practice in Nigeria

CMA: Comprehensive Meta-analysis Software

RR: Relative Risk

OD: Odds ratio

SSRI: Selective Serotonin Reuptake Inhibitor

PICOs: Participants, Intervention, Comparator, Outcomes

PRISMA: Preferred Reporting Items for Systematic Reviews and Meta-analysis

$\mathrm{NIH}$ : National Institute of Health

\section{Declarations}

\section{Ethics approval and consent to participate:}

The study will not require an ethical approval since it relies solely on secondary source of data (published studies).

\section{Consent for publication:}

Not applicable

Availability of data and materials:

Yes, data and materials are available

\section{Competing Interest:}

the authors declare that they have no competing interest.

\section{Support:}

\section{Funding:}

the project is funded by the Association for Good Clinical Practice in Nigeria AGCPN

The review work was conceived, supported and funded by the AGCPN.

Guarantor of the Review: 
DrEmmanuel Nna (e.nna@themping.orgm)

\section{Authors' contributions:}

EN and AFT conceived the project. EN, AFT, NA, AM, OO, OE and UA designed the study, NA, OO, AM will perform PubMed and AJOL searches, screen and review the articles; EN and OI will perform CINAHL and Embase search, OE and OM Cochrane Database and Web of science search while GOOGLE SCHOLAR , Scopus and Researchgate database search will be performed by KA, $\mathrm{OO}$ and $\mathrm{OI}$. Article handpicked and those obtained through contact with experts in the field will be equally screened by AFT.

\section{Acknowledgements:}

Thanks to Dr Sam Ibeneme who offered constructive guidance.

\section{Amendments:}

Important protocol amendments post registration will be recorded and included in dissemination.

\section{References}

1. Stewart DE. Depression during pregnancy. New England Journal of Medicine. 2011;365(17):1605-11.

2. Shakeel N, Eberhard-Gran M, Sletner L, Slinning K, Martinsen EW, Holme I, et al. A prospective cohort study of depression in pregnancy, prevalence and risk factors in a multi-ethnic population. BMC pregnancy and childbirth. 2015;15(1):5.

3. Rochat TJ, Tomlinson M, Bärnighausen T, Newell M-L, Stein A. The prevalence and clinical presentation of antenatal depression in rural South Africa. Journal of affective disorders. 2011;135(1-3):362-73.

4. Thompson O, Ajayi I. Prevalence of antenatal depression and associated risk factors among pregnant women attending antenatal clinics in Abeokuta North Local Government Area, Nigeria. Depression research and treatment. 2016;2016.

5. Ajinkya S, Jadhav PR, Srivastava NN. Depression during pregnancy: Prevalence and obstetric risk factors among pregnant women attending a tertiary care hospital in Navi Mumbai. Industrial psychiatry journal. 2013;22(1):37.

6. Koleva H, Stuart S, O'Hara MW, Bowman-Reif J. Risk factors for depressive symptoms during pregnancy. Archives of women's mental health. 2011;14(2):99-105.

7. Räisänen S, Lehto SM, Nielsen HS, Gissler M, Kramer MR, Heinonen S. Risk factors for and perinatal outcomes of major depression during pregnancy: a population-based analysis during 2002-2010 in Finland. BMJ open. 2014;4(11).

8. Aktas S, Calik KY. Factors affecting depression during pregnancy and the correlation between social support and pregnancy depression. Iranian Red Crescent Medical Journal. 2015;17(9). 
9. Schaffir J. Consequences of antepartum depression. Clinical Obstetrics and Gynecology. 2018;61(3):533-43.

10. Davalos DB, Yadon CA, Tregellas HC. Untreated prenatal maternal depression and the potential risks to offspring: a review. Archives of women's mental health. 2012;15(1):1-14.

11. Chaudron LH. Complex challenges in treating depression during pregnancy. American Journal of Psychiatry. 2013;170(1):12-20.

12. Pearson RM, Evans J, Kounali D, Lewis G, Heron J, Ramchandani PG, et al. Maternal depression during pregnancy and the postnatal period: risks and possible mechanisms for offspring depression at age 18 years. JAMA psychiatry. 2013;70(12):1312-9.

13. Chan J, Natekar A, Einarson A, Koren G. Risks of untreated depression in pregnancy. Canadian Family Physician. 2014;60(3):242-3.

14. Gentile S. Untreated depression during pregnancy: Short-and long-term effects in offspring. A systematic review. Neuroscience. 2017;342:154-66.

15. Kim DR, O'Reardon JP, Epperson CN. Guidelines for the management of depression during pregnancy. Current psychiatry reports. 2010;12(4):279-81.

16. Croen LA, Grether JK, Yoshida CK, Odouli R, Hendrick V. Antidepressant use during pregnancy and childhood autism spectrum disorders. Archives of general psychiatry. 2011;68(11):1104-12.

17. Boukhris T, Sheehy O, Mottron L, Bérard A. Antidepressant use during pregnancy and the risk of autism spectrum disorder in children. JAMA pediatrics. 2016;170(2):117-24.

18. Molenaar NM, Kamperman AM, Boyce P, Bergink V. Guidelines on treatment of perinatal depression with antidepressants: An international review. Australian \& New Zealand Journal of Psychiatry. 2018;52(4):320-7.

19. Byatt N, Deligiannidis KM, Freeman MP. Antidepressant use in pregnancy: a critical review focused on risks and controversies. Acta Psychiatrica Scandinavica. 2013;127(2):94-114.

20. Kornum JB, Nielsen RB, Pedersen L, Mortensen PB, Nørgaard M. Use of selective serotonin-reuptake inhibitors during early pregnancy and risk of congenital malformations: updated analysis. Clinical Epidemiology. 2010;2:29.

21. Gao S-Y, Wu Q-J, Sun C, Zhang T-N, Shen Z-Q, Liu C-X, et al. Selective serotonin reuptake inhibitor use during early pregnancy and congenital malformations: a systematic review and meta-analysis of cohort studies of more than 9 million births. BMC medicine. 2018;16(1):1-14.

22. Huybrechts KF, Palmsten K, Avorn J, Cohen LS, Holmes LB, Franklin JM, et al. Antidepressant use in pregnancy and the risk of cardiac defects. New England Journal of Medicine. 2014;370(25):2397407.

23. Jimenez-Solem E, Andersen JT, Petersen M, Broedbaek K, Jensen JK, Afzal S, et al. Exposure to selective serotonin reuptake inhibitors and the risk of congenital malformations: a nationwide cohort study. BMJ open. 2012;2(3). 
24. Moher D, Shamseer L, Clarke M, Ghersi D, Liberati A, Petticrew M, et al. Preferred reporting items for systematic review and meta-analysis protocols (PRISMA-P) 2015 statement. Systematic reviews. 2015;4(1):1.

\section{Supplementary Files}

This is a list of supplementary files associated with this preprint. Click to download.

- PRISMAPchecklistBMCSSRIstudy.docx

- PRISMAPchecklistBMCSSRIstudy.docx 